\title{
TIME VARIATION OF THE ELECTRON MASS IN THE EARLY UNIVERSE AND THE BARROW-MAGUEIJO MODEL
}

\author{
Claudia G. Scóccola, ${ }^{1,2,3}$ Mercedes E. Mosquera, ${ }^{1,2}$ Susana J. Landau, ${ }^{1,4,5}$ and Héctor Vucetich ${ }^{1,5}$ \\ Received 2007 September 13; accepted 2008 March 2
}

\begin{abstract}
We put limits on the time variation of the electron mass in the early universe using observational primordial abundances of $\mathrm{D},{ }^{4} \mathrm{He}$, and ${ }^{7} \mathrm{Li}$, recent data from the cosmic microwave background, and the $2 \mathrm{dFGRS}$ power spectrum. Furthermore, we use these constraints together with other astronomical and geophysical bounds from the late universe to test the Barrow-Magueijo model for the variation in $m_{e}$. From our analysis we obtain $-0.615<G \omega / c^{4}<$ -0.045 ( $3 \sigma$ interval), in disagreement with the result obtained in the original paper.
\end{abstract}

Subject headings: cosmic microwave background - cosmology: theory — early universe

\section{INTRODUCTION}

Time variation of fundamental constants over cosmological timescales is a prediction of theories that attempt to unify all fundamental interactions, such as string-derived field theories $(\mathrm{Wu}$ \& Wang 1986; Maeda 1988; Barr \& Mohapatra 1988; Damour \& Polyakov 1994; Damour et al. 2002a, 2002b), related braneworld theories (Youm 2001a, 2001b; Palma et al. 2003; Brax et al. 2003), and Kaluza-Klein theories (Kaluza 1921; Klein 1926; Weinberg 1983; Gleiser \& Taylor 1985; Overduin \& Wesson 1997). In order to study the possible variation in the fine-structure constant or the electron mass, theoretical frameworks based on first principles were developed by different authors (Bekenstein 1982, 2002; Barrow et al. 2002; Barrow \& Magueijo 2005).

The predicted time behavior of the fundamental constants depends on which version of the theories is considered. Thus, bounds obtained from astronomical and geophysical data are an important tool to test the validity of these theories. In a previous work (Mosquera et al. 2008), we have analyzed the variation in the fine-structure constant in the context of the Bekenstein model. In this paper, instead, we study the variation in the electron mass $\left(m_{e}\right)$ in the context of the Barrow-Magueijo model (Barrow \& Magueijo 2005). Note that $m_{e}$ is not a fundamental constant in the same sense as the fine-structure constant. Hence, it could be argued that constraints on the time variation of the Higgs vacuum expectation value $(\langle v\rangle)$, rather than $m_{e}$, are more relevant. Moreover, the possibility of a time variation of the vacuum expectation value of a field seems more plausible than the time variation of a gauge coupling constant. However, in the context of the Barrow-Magueijo model, the relevant fundamental constant is $m_{e}$, and thus we focus on its possible variation. The joint variation in the fine-structure constant and $\langle v\rangle$ in the early universe will be analyzed in a forthcoming paper.

Constraints on $m_{e}$ variation over cosmological timescales are available from astronomical and local methods. The latter include geophysical methods (analysis of natural long-lived $\beta$-decayers in geological minerals and meteorites) and laboratory measurements (comparisons of different transitions in atomic clocks). The astronomical methods are based mainly on the analysis of spectra

\footnotetext{
${ }^{1}$ Facultad de Ciencias Astronómicas y Geofísicas, Universidad Nacional de La Plata, Paseo del Bosque S/N, 1900 La Plata, Argentina.

${ }^{2}$ Fellow of CONICET.

3 Also at Instituto de Astrofísica La Plata.

${ }^{4}$ Departamento de Física, FCEyN, Universidad de Buenos Aires, Ciudad Universitaria-Pab. 1, 1428 Buenos Aires, Argentina.

5 Member of the Carrera del Investigador Científico y Tecnológico, CONICET.
}

from high-redshift quasar absorption systems. Bounds on the variation in $m_{e}$ in the early universe can be obtained using data from the cosmic microwave background (CMB) radiation and from the abundances of light elements. These bounds are not as stringent as those mentioned above, but they are important because they refer to a different cosmological epoch.

In this paper we perform a careful study of the time variation of $m_{e}$ in the early universe. First, we use all available abundances of $\mathrm{D},{ }^{4} \mathrm{He}$, and ${ }^{7} \mathrm{Li}$, the latest data from the CMB and the Two Degree Field Galaxy Redshift Survey (2dFGRS) power spectrum to put bounds on the variation in $m_{e}$ without assuming any theoretical model. Afterward, we use these bounds and others from astronomical and geophysical data to test Barrow-Magueijo theory.

In $\S 2$ we use the abundances of the light elements to put bounds on $\Delta m_{e} /\left(m_{e}\right)_{0}$, where $\left(m_{e}\right)_{0}$ is the present value of $m_{e}$, allowing the baryon to photon density $\eta_{B}$ to vary. In $\S 3$ we use the 3 year Wilkinson Microwave Anisotropy Probe (WMAP) data, other CMB experiments and the power spectrum of the final 2dFGRS to put bounds on the variation in $m_{e}$ during recombination, allowing also other cosmological parameters to vary. In $\S \S 4,5$, and 6 we describe the astronomical and local data from the late universe. In $\S 7$ we describe the Barrow-Magueijo model and obtain solutions for the scalar field that drives the variation in $m_{e}$, for the early and late universe. In $\S 8$ we show our results. Finally, in $\S 9$ we discuss the results and summarize our conclusions.

\section{BOUNDS FROM BBN}

Big bang nucleosynthesis (BBN) is one of the most important tools for studying the early universe. The baryon-to-photon ratio $\eta_{B}$ or equivalently the baryon asymmetry $\eta_{B} \equiv n_{B}-n_{\bar{B}} / n_{\gamma}$ can be determined by comparison between theoretical calculations and observations of the abundances of light elements. An independent method for determining $\eta_{B}$ is provided by data from the CMB (Spergel et al. 2003, 2007; Sánchez et al. 2006). Considering the baryon density from WMAP results, the predicted abundances are highly consistent with the observed D, but not with all ${ }^{4} \mathrm{He}$ and ${ }^{7} \mathrm{Li}$. Such a discrepancy is usually ascribed to nonreported systematic errors in the observations of ${ }^{4} \mathrm{He}$ and ${ }^{7} \mathrm{Li}$. However, if the systematic errors of ${ }^{4} \mathrm{He}$ and ${ }^{7} \mathrm{Li}$ are correctly estimated, it may allow us to gain insight into new physics beyond the minimal BBN model.

In the currently most popular particle physics models, the lepton and baryon numbers are comparable. In this case, any asymmetry between neutrinos and antineutrinos will have no noticeable effect 
TABLE 1

Best-Fit Parameter Values and $1 \sigma$ Errors for the BBN Constraints on $\Delta m_{e} /\left(m_{e}\right)_{0}$ AND $\eta_{B}$

\begin{tabular}{|c|c|c|c|}
\hline Data Set & $\eta_{B} \pm \sigma^{\mathrm{a}}$ & $\Delta m_{e} /\left(m_{e}\right)_{0} \pm \sigma$ & $\chi_{\min }^{2} /(N-2)$ \\
\hline $\mathrm{D}+{ }^{4} \mathrm{He}+{ }^{7} \mathrm{Li} \ldots \ldots \ldots \ldots$ & $4.237_{-0.097}^{+0.047}$ & $-0.036_{-0.007}^{+0.010}$ & 9.33 \\
\hline${ }^{4} \mathrm{He}+{ }^{7} \mathrm{Li} \ldots \ldots \ldots \ldots \ldots \ldots$ & $3.648_{-0.124}^{+0.128}$ & $-0.055_{-0.008}^{+0.010}$ & 1.00 \\
\hline $\mathrm{D}+{ }^{7} \mathrm{Li} \ldots \ldots$. & $5.399_{-0.213}^{+0.284}$ & $0.653_{-0.045}^{+0.008}$ & 1.01 \\
\hline $\mathrm{D}+{ }^{4} \mathrm{He} \ldots \ldots$. & $6.339_{-0.355}^{+0.376}$ & $-0.022 \pm 0.009$ & 1.01 \\
\hline
\end{tabular}

${ }^{\text {a }}$ Units of $10^{-10}$.

on the predictions of BBN. However, observational data do not imply that the lepton asymmetry should be connected to the "tiny" baryon asymmetry $\eta_{B}$. Moreover, a small asymmetry between electron-type neutrinos and antineutrinos can have a significant impact on BBN, since the $\nu_{e}$ affect the interconversion of neutrons to protons, changing the equilibrium neutron-to-proton ratio from $(n / p)_{\mathrm{eq}}^{0}=e^{-(\Delta m / T)}$ to $(n / p)_{\mathrm{eq}}=(n / p)_{\mathrm{eq}}^{0} e^{-\xi_{e}}$, where $\xi_{e}$ is the ratio of the neutrino chemical potential to the temperature. Consequently, the ${ }^{4} \mathrm{He}$ abundance changes. In contrast, the D abundance is insensitive to $\xi_{e} \neq 0$. Consistent with the BBN and CMB data, values of $\xi_{e}$ in the range $-0.1<\xi_{e}<0.3$ are permitted (Barger et al. 2003; Steigman 2005, 2006). In this work, however, we assume $\xi_{e} \simeq 0$ and attribute the discrepancies described above to the time variation of $m_{e}$ or $\langle v\rangle$.

We considered available observational data on $\mathrm{D},{ }^{4} \mathrm{He}$, and ${ }^{7} \mathrm{Li}$. For D, we used the values reported by Pettini \& Bowen (2001), O’Meara et al. (2001), Kirkman et al. (2003), Burles \& Tytler (1998a, 1998b), Crighton et al. (2004), O’Meara et al. (2006), and Oliveira et al. (2006). For ${ }^{4} \mathrm{He}$, the available observations are reported by Peimbert et al. (2007) and Izotov et al. (2007). For ${ }^{7} \mathrm{Li}$, we considered the results from Ryan et al. (2000), Bonifacio et al. (2002, 2007), Bonifacio \& Molaro (1997), Molaro et al. 1997, Asplund et al. (2006), and Boesgaard et al. (2005). For the discussion about the consistency data check, we refer the reader to an earlier work (Mosquera et al. 2008).

We modified the numerical code of Kawano $(1988,1992)$ in order to allow $m_{e}$ to vary. The code was also updated with the reaction rates reported by Bergström et al. (1999). The main effects of the possible variation in $m_{e}$ in the physics of the first 3 minutes of the universe are changes in the weak rates, in the sum of electron and positron energy densities, in the sum of electron

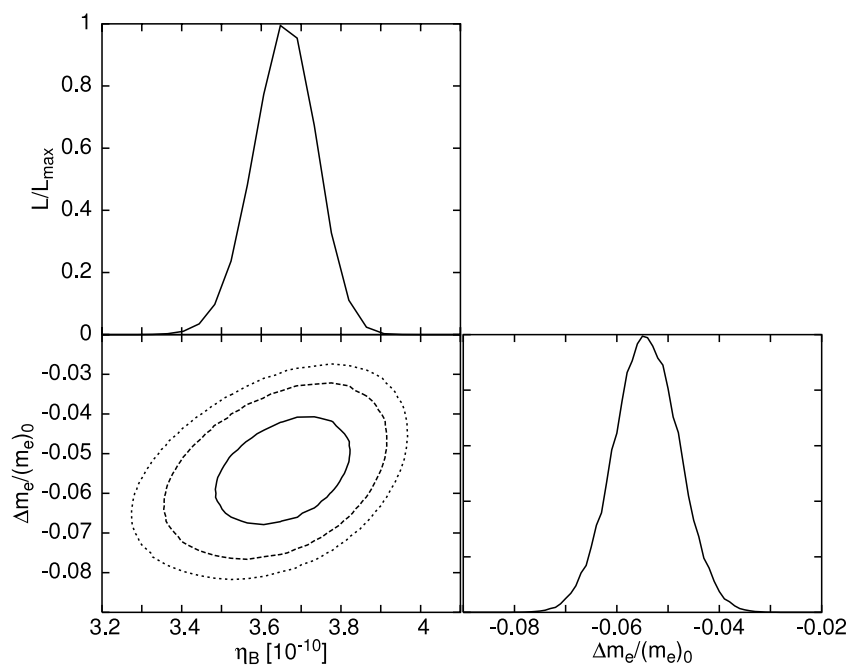

and positron pressures, and in the difference of electron and positron number densities (see Appendix A for details).

If $m_{e}$ takes a lower value than the present one, the primordial abundances are higher than the standards. The change is more important for ${ }^{4} \mathrm{He}$ and ${ }^{7} \mathrm{Li}$ abundances, where a variation of $10 \%$ in $m_{e}$ leads to a change of $7.4 \%$ and $8.5 \%$ in the abundances, while the effect on the D abundance is tiny (1.5\%).

We computed the light nuclei abundances for different values of $\eta_{B}$ and $\Delta m_{e} /\left(m_{e}\right)_{0}$ and performed the statistical analysis to obtain the best-fit values for these parameters. There is no good fit for the whole data set even for $\Delta m_{e} /\left(m_{e}\right)_{0} \neq 0$. However, reasonable fits can be found excluding one group of data at each time (see Table 1). Figures 1 and 2 show the confidence contours and one-dimensional likelihoods for different groups of data.

We obtained the result that for $\mathrm{D}+{ }^{4} \mathrm{He}$ the value of $\eta_{B}$ is coincident with WMAP estimation and there is no variation in $m_{e}$ within $3 \sigma$. Moreover, the other groups of data favor values far from $W M A P$ estimation, and for $\mathrm{D}+{ }^{7} \mathrm{Li}$ the result is consistent with variation in $m_{e}$ within $6 \sigma$.

As pointed out in the introduction, in the standard model D, ${ }^{4} \mathrm{He}$, and ${ }^{7} \mathrm{Li}$ abundances considered separately predict very different values for the baryon density. Therefore, when the three abundances are fitted together, an intermediate value of $\eta_{B}$ is obtained, but the value of $\chi^{2}$ is too high. Only when two abundances are considered do we obtain a reasonable fit. Furthermore, a high variation in $m_{e}$ which affects mostly the ${ }^{7} \mathrm{Li}$ abundance, is needed to fit $\mathrm{D}$ and ${ }^{7} \mathrm{Li}$ together. On the other hand, D and ${ }^{4} \mathrm{He}$ are marginally consistent with the WMAP estimation, and therefore no variation in $m_{e}$ is needed to fit both data at the same time. Finally, in order to fit the abundances of ${ }^{4} \mathrm{He}$ and ${ }^{7} \mathrm{Li}$, a variation in $m_{e}$ is needed, since both quantities are affected when $m_{e}$ is allowed to vary.

As mentioned in the introduction, in this paper we limit ourselves to the context of the Barrow-Magueijo model of a varying $m_{e}$. However, in more general classes of theories (Kaluza-Klein, strings, GUTs, etc.), the underlying fundamental constant is the Higgs vacuum expectation value. The dependence of the primordial abundances on the Higgs vacuum expectation value has been analyzed by Yoo \& Scherrer (2003). Semianalytical analysis have been performed by some of us in earlier works (Chamoun et al. 2007). Besides changes in $m_{e}$, a possible variation in $\langle v\rangle$ modifies the values of the following quantities: the Fermi constant $G_{\mathrm{F}}$, the neutron-proton mass difference $\Delta m_{\mathrm{np}}$, and the deuterium

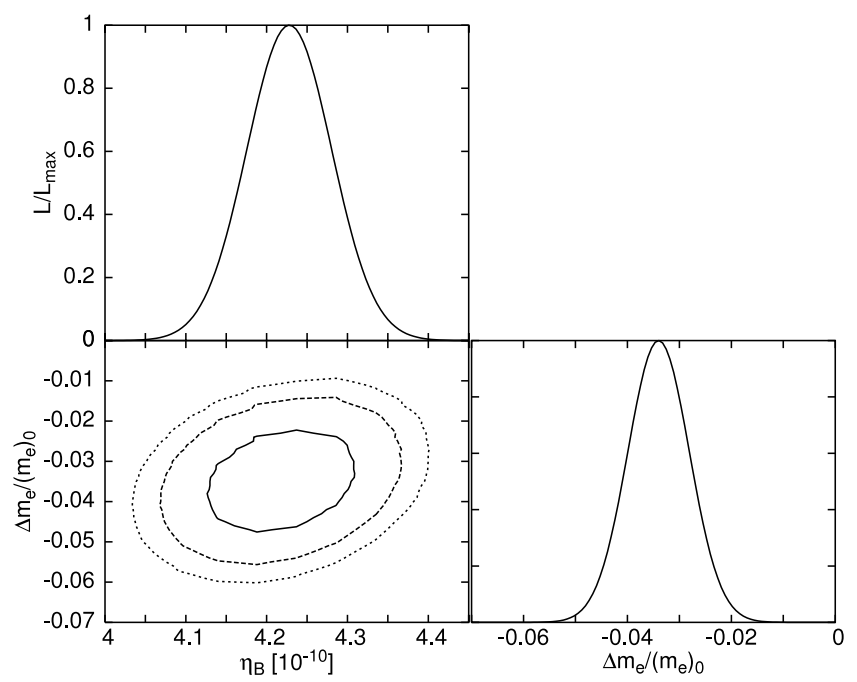

FIG. 1.-The 1, 2, and $3 \sigma$ likelihood contours for $\Delta m_{e} /\left(m_{e}\right)_{0}$ vs. $\eta_{B}$ and one-dimensional likelihood using ${ }^{4} \mathrm{He}+{ }^{7}$ Li data $(l e f t)$ and all data $(r i g h t)$. 

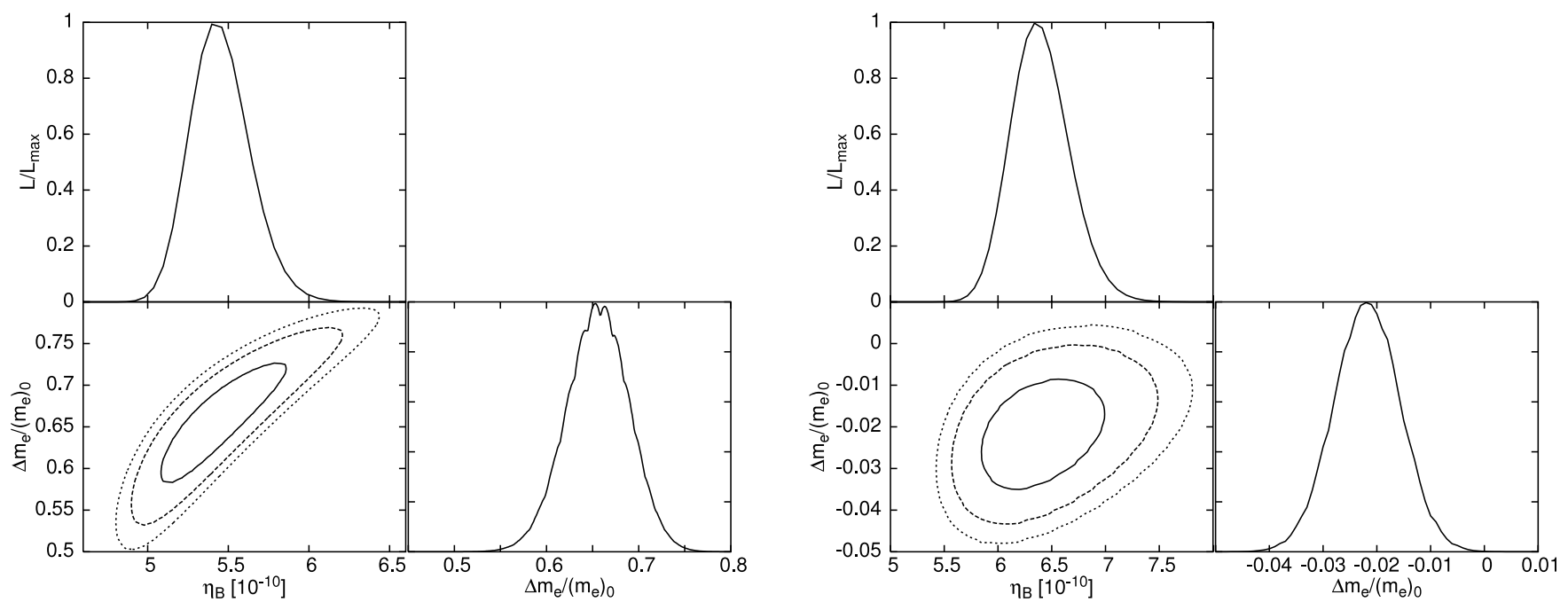

FIG. 2.- Same as Fig. 1, but using D $+{ }^{7}$ Li data (left) and $\mathrm{D}+{ }^{4} \mathrm{He}$ (right).

binding energy $\epsilon_{\mathrm{D}}$. The dependence of these quantities with $\langle v\rangle$ have been described in an earlier work (Chamoun et al. 2007) (see Appendix A for details). We modified numerical code of Kawano in order to allow $\langle v\rangle$ to vary.

The abundances of the primordial elements are much higher than the standard value if the Higgs vacuum expectation value during $\mathrm{BBN}$ is larger than the current value. A variation of $10 \%$ in $\langle v\rangle$ leads to a change of $45 \%, 25 \%$, and $29 \%$ in the ${ }^{4} \mathrm{He},{ }^{7} \mathrm{Li}$, and $\mathrm{D}$ abundances, respectively. Since $\mathrm{D}$ is a residual of ${ }^{4} \mathrm{He}$ production, a great change in ${ }^{4} \mathrm{He}$ also leads to an important change in $\mathrm{D}$. The changes in the abundances are greater than in the case where only $m_{e}$ is allowed to vary.

In the case of $\langle v\rangle$, we performed the same analysis for the same groups of data we considered for $m_{e}$. As in the case of $m_{e}$, variation there is no good fit for the whole set of data. However, reasonable fits can be found excluding one group of data at each time (see Table 2). Figure 3 shows the confidence contours and one-dimensional likelihoods for different groups of data.

We obtain the result that for $\mathrm{D}+{ }^{4} \mathrm{He}$ the value of $\eta_{B}$ is consistent with $W M A P$ estimation and there is no variation in $\langle v\rangle$ within $3 \sigma$. Moreover, the other groups of data favor values that are not consistent with $W M A P$ results. For $\mathrm{D}+{ }^{7} \mathrm{Li}$, the result is consistent with variation in $\langle v\rangle$ within $6 \sigma$. The results are similar to those obtained in the case where $m_{e}$ is the varying constant: (1) no reasonable fit exists for the three abundances; (2) D and ${ }^{4} \mathrm{He}$ can be well fitted with null $\langle v\rangle$ variation; (3) huge variation in $\mathrm{D}$ and ${ }^{7} \mathrm{Li}$ is required to obtain a reasonable fit. However, the bounds on variation in $\langle v\rangle$ are more stringent than the bounds obtained when only $m_{e}$ was allowed to vary (see Table 1$)$. This could be explained by the fact that variations in $\langle v\rangle$ lead to greater changes in the theoretical abundances than variation in $m_{e}$.

TABLE 2

Best-Fit Parameter Values and $1 \sigma$ Errors for the BBN Constraints on $\Delta\langle v\rangle /\langle v\rangle_{0}$ AND $\eta_{B}$

\begin{tabular}{|c|c|c|c|}
\hline Data Set & $\eta_{B} \pm \sigma^{\mathrm{a}}$ & $\Delta\langle v\rangle /\langle v\rangle_{0} \pm \sigma$ & $\chi_{\min }^{2} /(N-2)$ \\
\hline $\mathrm{D}+{ }^{4} \mathrm{He}+{ }^{7} \mathrm{Li}$. & $4.275 \pm 0.097$ & $0.006 \pm 0.002$ & 9.27 \\
\hline${ }^{4} \mathrm{He}+{ }^{7} \mathrm{Li} \ldots \ldots \ldots \ldots \ldots$ & $3.723_{-0.124}^{+0.132}$ & $0.008 \pm 0.001$ & 1.00 \\
\hline $\mathrm{D}+{ }^{7} \mathrm{Li} \ldots \ldots \ldots \ldots \ldots \ldots$ & $5.139_{-0.231}^{+0.124}$ & $-0.138_{-0.009}^{+0.015}$ & 1.01 \\
\hline $\mathrm{D}+{ }^{4} \mathrm{He} . \ldots \ldots \ldots \ldots \ldots \ldots \ldots$ & $6.324_{-0.285}^{+0.371}$ & $0.004 \pm 0.002$ & 1.04 \\
\hline
\end{tabular}

${ }^{\text {a }}$ Units of $10^{-10}$.

\section{BOUNDS FROM THE COSMIC MICROWAVE BACKGROUND}

$\mathrm{CMB}$ radiation provides valuable information about the physical conditions of the universe just before the decoupling of matter and radiation, and thanks to its dependence on cosmological parameters, it allows for them to be estimated. Any change in the value of $m_{e}$ affects the physics during recombination, mainly the redshift of this epoch, due to a shift in the energy levels and, in particular, the binding energy of hydrogen. The Thompson scattering cross section, which is proportional to $m_{e}^{-2}$, is also changed for all particles. Therefore, the CMB power spectrum is modified by a change in the relative amplitudes of the Doppler peaks and shifts in their positions (see Appendix B for details). Changes in the cosmological parameters produce similar effects. In the recombination scenario the only effect of varying $\langle v\rangle$ is a change in the value of $m_{e}$. Previous analysis of the CMB data including a possible variation in $m_{e}$ have been performed by Yoo \& Scherrer (2003) and Kujat \& Scherrer (2000). In this paper we use the $W M A P 3$ year temperature and temperature-polarization power spectrum (Spergel et al. 2007) and other CMB experiments, such as CBI (Readhead et al. 2004), ACBAR (Kuo et al. 2004), and BOOMERANG (Piacentini et al. 2006; Jones et al. 2006), and the power spectrum of the $2 \mathrm{dFGRS}$ (Cole et al. 2005). We consider a spatially flat cosmological model with adiabatic density fluctuations. The parameters of our model are

$$
P=\left[\Omega_{B} h^{2}, \Omega_{\mathrm{CDM}} h^{2}, \Theta, \tau_{r e}, \frac{\Delta m_{e}}{\left(m_{e}\right)_{0}}, n_{s}, A_{s}\right],
$$

where $\Omega_{\mathrm{CDM}} h^{2}$ is the dark matter density in units of the critical density, $\Theta$ gives the ratio of the comoving sound horizon at decoupling to the angular diameter distance to the surface of last scattering, $\tau_{r e}$ is the reionization optical depth, $n_{s}$ the scalar spectral index, and $A_{s}$ is the amplitude of the density fluctuations.

We use a Markov chain Monte Carlo method to explore the parameter space because the exploration of a multidimensional parameter space with a grid of points is computationally prohibitive. We use the publicly available CosmoMC code of Lewis \& Bridle (2002), which uses CAMB (Lewis et al. 2000) and RECFAST (Seager et al. 1999) to compute the CMB power spectra, and we have modified them in order to include the possible variation in $m_{e}$ at recombination. We ran eight different chains. We used the convergence criterion of Raftery \& Lewis (1992) to stop the chains 

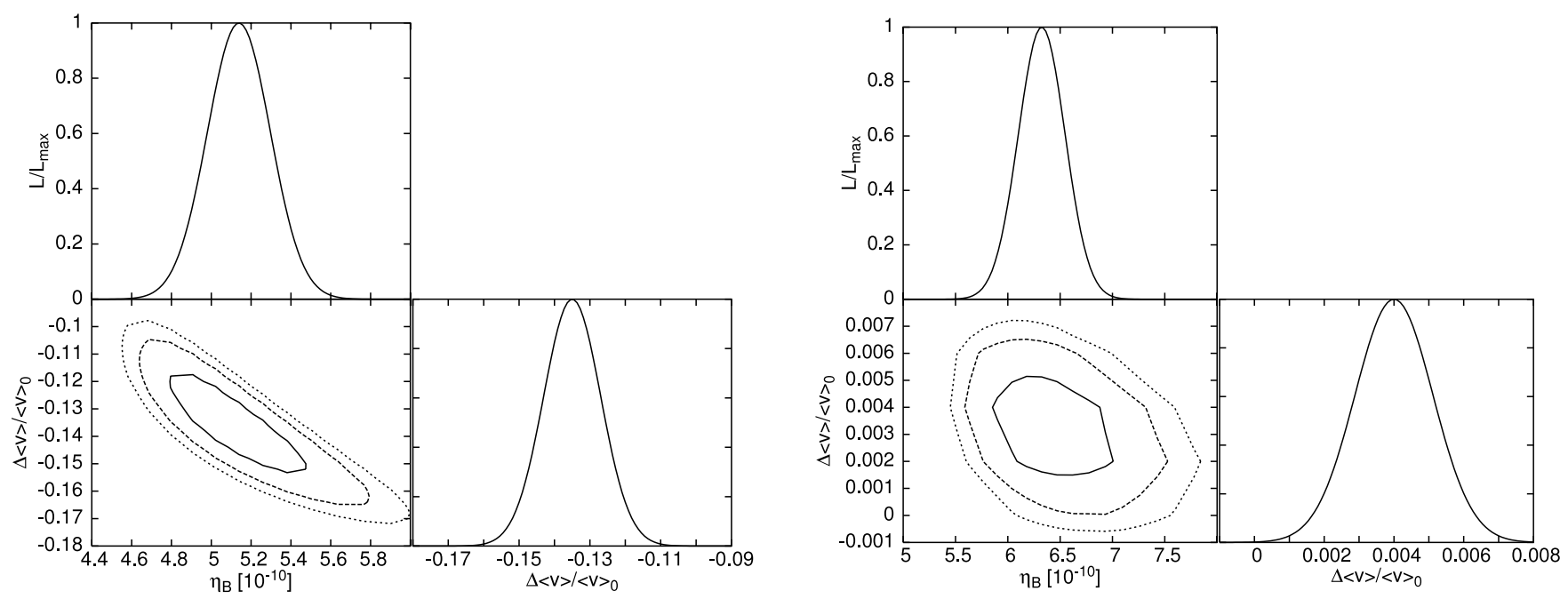

FIG. 3. - Same as Fig. 1, but for $\Delta\langle v\rangle /\langle v\rangle_{0}$ vs. $\eta_{B}$ using $\mathrm{D}+{ }^{7} \mathrm{Li}($ left $)$ and $\mathrm{D}+{ }^{4} \mathrm{He}(r i g h t)$.

when $R-1<0.0044$ (which is more stringent than the usually adopted value). Results are shown in Table 3 and Figure 4. Figure 4 shows a strong degeneracy between $m_{e}$ and $\Theta$, which is directly related to $H_{0}$, and also between $m_{e}$ and $\Omega_{B} h^{2}$, and $m_{e}$ and $\Omega_{\mathrm{CDM}} h^{2}$.

We have also performed the analysis considering only $\mathrm{CMB}$ data. The strong degeneracy between $m_{e}$ and $H_{0}$ made the chains move along the entire wide $H_{0}$ prior, making it impossible to find reliable mean values and errors. Hence, we added a Gaussian prior to $H_{0}$, which was obtained from the Hubble Space Telescope Key Project (Freedman et al. 2001), and chose the values of the mean and errors as those inferred from the closest objects in that paper, so we could neglect any possible difference between the value of $m_{e}$ at that redshift and the present value. In this way, we postprocessed the chains and found limits that are consistent with those of the first analysis, revealing the robustness of these bounds. However, the most stringent constraints were obtained in the first analysis (see Fig. 5).

Finally, we comment that Planck will be the first mission to map the entire CMB sky with millijansky sensitivity and resolution better than $10^{\prime}$ ( Villa et al. 2006). This resolution will make it possible to see into the damping tail of the anisotropy spectrum, around the third and fourth peaks, with a precision limited almost solely by cosmic variance (White 2006). This will allow for a very precise estimation of the baryon-to-photon ratio from the relative height of the peaks in the spectrum.

\section{BOUNDS FROM QUASAR ABSORPTION SYSTEMS}

Quasar absorption systems present ideal laboratories in which to search for any temporal variation of the fundamental constants over cosmological timescales. In particular, a method for constraining the variation in $\mu=m_{p} / m_{e}$ was developed by Varshalovich \& Levshakov (1993). It is based on the fact that wavelengths of electron-vibro-rotational lines depend on the reduced mass of the molecules, with different dependence for different transitions. In this way, it is possible to distinguish the cosmological redshift of a line from the shift caused by a variation in $\mu$. The rest-frame laboratory wavelength, $\lambda_{i}^{0}$, can be related to those in the quasar absorption system, $\lambda_{i}$, as $\lambda_{i} / \lambda_{i}^{0}=\left(1+z_{\text {abs }}\right)\left[1+K_{i}(\Delta \mu / \mu)\right]$, where $z_{\text {abs }}$ is the absorption redshift and $K_{i}$ is the coefficient that determines the sensitivity of the wavelength $\lambda_{i}$. Using observations from $\mathrm{H}_{2}$ absorption systems at high redshift and laboratory measurements, several authors obtained constraints on $\mu$ (Potekhin et al. 1998; Levshakov et al. 2002; Ivanchik et al. 2003, 2005). The most up-to-date available measurements for each redshift are listed in Table 4 and are those that we consider in order to test Barrow-Magueijo model.

Another method for constraining variation in fundamental constants is based on the comparison between the hyperfine $21 \mathrm{~cm}$ absorption transition of neutral hydrogen $\left(\nu_{a}\right)$ and an optical resonance transition $\left(\nu_{b}\right)$. The ratio $\nu_{a} / \nu_{b}$ is proportional to $x=$ $\alpha^{2} g_{p}\left(m_{e} / m_{p}\right)$, where $g_{p}$ is the proton $g$ factor (Tzanavaris et al. 2007). Thus, a change of this quantity will result in a difference in the redshift measured from $21 \mathrm{~cm}$ and optical absorption lines as $\Delta x / x=\left(z_{\text {opt }}-z_{21}\right) /\left(1+z_{21}\right)$. Since we are working in the context of the Barrow \& Magueijo (2005) model, the only fundamental constant that is allowed to vary is $m_{e}$. Table 5 shows the bounds obtained by Tzanavaris et al. (2007) combining the measurements of optical and radio redshift. This method has the inconvenience that it is difficult to determine if both radio and

TABLE 3

Mean Values and Errors for the Main and Derived Parameters including $m_{e}$ Variation

\begin{tabular}{|c|c|c|c|}
\hline Parameter & Mean Value and $1 \sigma$ Error & Parameter & Mean Value and $1 \sigma$ Error \\
\hline$\Omega_{B} h^{2} \ldots \ldots \ldots \ldots \ldots \ldots$ & $0.0217 \pm 0.0010$ & $\Omega_{\mathrm{CDM}} h^{2} \ldots$ & $0.1006_{-0.0086}^{+0.0085}$ \\
\hline$\Theta$ & $1.020 \pm 0.025$ & $\tau_{\text {re }} \ldots \ldots \ldots \ldots \ldots \ldots \ldots \ldots$ & $0.091_{-0.014}^{+0.013}$ \\
\hline$\Delta m_{e} /\left(m_{e}\right)_{0} \ldots \ldots \ldots \ldots \ldots$ & $-0.029 \pm 0.034$ & $n_{s} \ldots \ldots \ldots \ldots \ldots \ldots \ldots \ldots$ & $0.960 \pm 0.015$ \\
\hline$A_{s} \ldots \ldots \ldots \ldots \ldots \ldots \ldots \ldots \ldots$ & $3.020 \pm 0.064$ & $H_{0}^{\mathrm{a}} \ldots \ldots \ldots \ldots \ldots \ldots \ldots$ & $68.1_{-6.0}^{+5.9}$ \\
\hline
\end{tabular}

${ }^{\mathrm{a}}$ Units of $\mathrm{km} \mathrm{s}^{-1} \mathrm{Mpc}^{-1}$. 


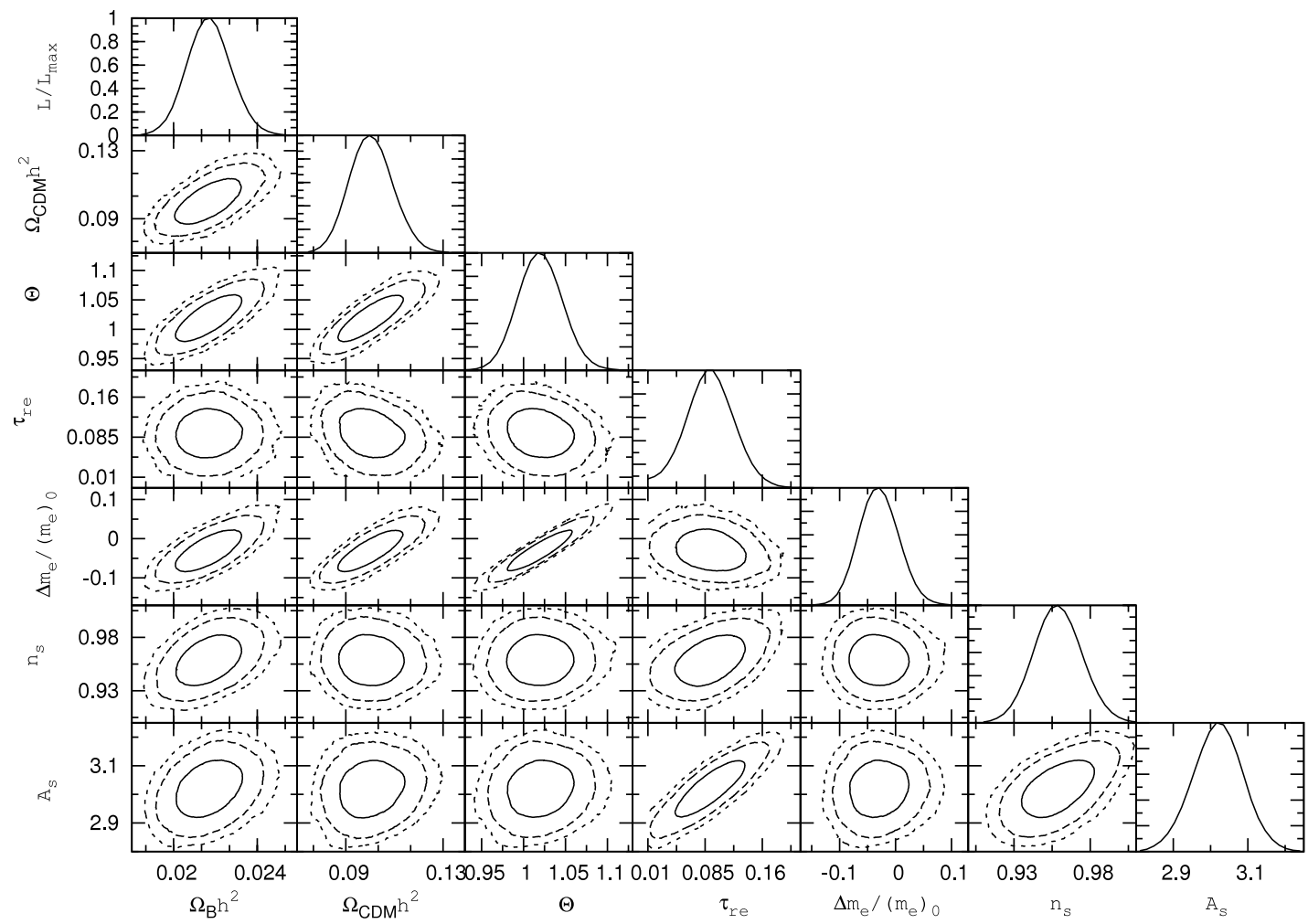

FIG. 4.-Marginalized posterior distributions obtained with CMB data, including the WMAP 3 year data release plus 2dFGRS power spectrum. The diagonal shows the posterior distributions for individual parameters, the other panels shows the two-dimensional contours for pairs of parameters, marginalizing over the others.

optical lines originated at the same absorption system. Thus, a difference in the velocity of the absorption clouds could hide a variation in $x$.

\section{BOUNDS FROM GEOPHYSICAL DATA}

The half-life of long-lived $\beta$-decayers has been determined both in laboratory measurements and by comparison with the age of meteorites, as found from $\alpha$-decay radioactivity analysis. The most stringent bound on the variation in the half life, $\lambda$, proceeds from the comparison of ${ }^{187} \mathrm{Re}$-decay during the formation of the solar system and in the present (Olive et al. 2004): $\Delta \lambda / \lambda=$ $-0.016 \pm 0.016$. Sisterna \& Vucetich (1990) derived a relation between the shift in the half-life of long-lived $\beta$-decayers and a possible variation in the values of the fundamental constants over cosmological time. In this paper we only consider $m_{e}$ varia-

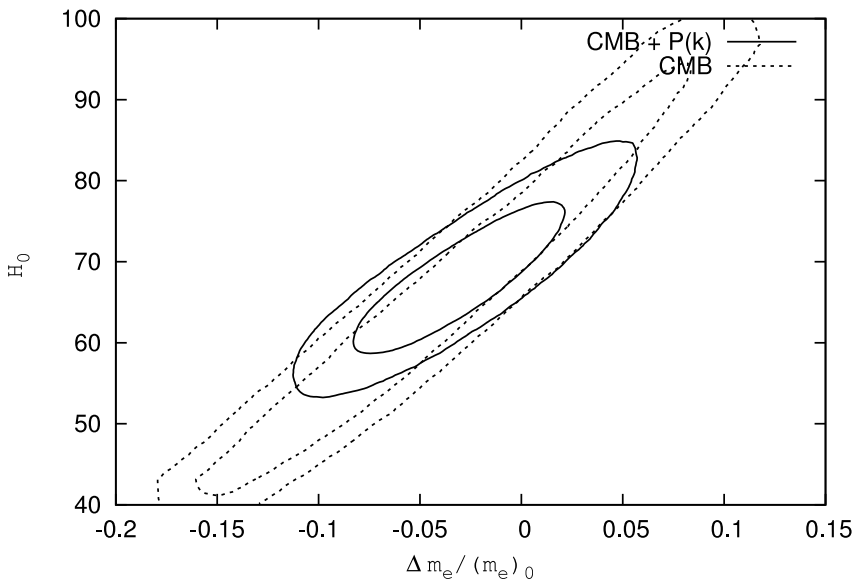

tion and therefore $\Delta \lambda / \lambda=a\left[\Delta m_{e} /\left(m_{e}\right)_{0}\right]$, where $a=-600$ for ${ }^{187} \mathrm{Re}$.

\section{BOUNDS FROM LABORATORY}

Comparing the frequencies of selected atomic transitions over time is a useful tool for putting stringent bounds on the variation in the fundamental constants at present. In particular, the hyperfine frequency of cesium can be approximated by $\nu_{\mathrm{Cs}} \simeq g_{\mathrm{Cs}}\left(m_{e} /\right.$ $\left.m_{p}\right) \alpha^{2} \mathrm{R}_{y} F_{\mathrm{Cs}}(\alpha)$ [where $g_{\mathrm{Cs}}$ is the nuclear $g$ factor, $\mathrm{R}_{y}$ is the Rydberg constant expressed as a frequency, and $F_{\mathrm{Cs}}(\alpha)$ is a dimensionless function of $\alpha$ and does not depend on $m_{e}$ at least at first order], while optical transition frequencies can be expressed as $\nu_{\mathrm{opt}} \simeq \mathrm{R}_{y} F(\alpha)$. Several authors (Bize et al. 2003; Fischer et al. 2004; Peik et al. 2004) have measured various optical transitions and compared them with the frequency of the ground-state

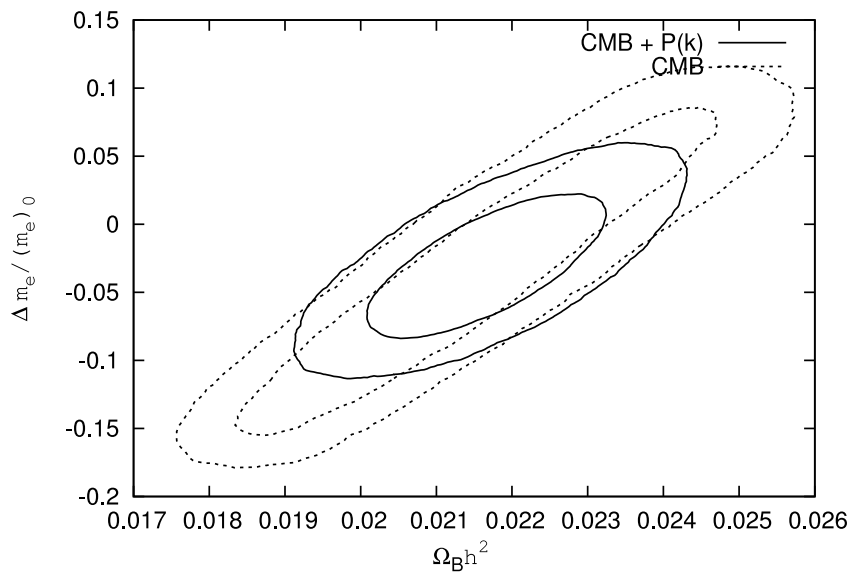

FIG. 5.-The 1 and $2 \sigma$ confidence level contours obtained with CMB data with and without data of the 2dFGRS power spectrum. 
TABLE 4

Absorption Redshift and Value of $\Delta m_{e} /\left(m_{e}\right)_{0}$ Obtained Comparing Molecular and Laboratory Wavelengths

\begin{tabular}{|c|c|c|}
\hline Redshift & $\Delta m_{e} /\left(m_{e}\right)_{0} \pm \sigma^{\mathrm{a}}$ & Reference \\
\hline 2.8. & $-6.25 \pm 13.70$ & Potekhin et al. (1998) \\
\hline 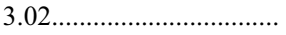 & $-1.40 \pm 0.83$ & Ivanchik et al. (2005) \\
\hline $2.6 \ldots$ & $-2.11 \pm 1.39$ & Ivanchik et al. (2005) \\
\hline
\end{tabular}

${ }^{\mathrm{a}}$ In units of $10^{-5}$.

hyperfine splitting in neutral ${ }^{133} \mathrm{Cs}$. These measurements can be used to constrain the variation in $\dot{m}_{e} /\left(m_{e}\right)_{0}$. Constraints from different experiments are listed in Table 6.

\section{THE MODEL}

We now analyze the Barrow-Magueijo model for the variation in $m_{e}$. We solve the equation of the scalar field $(\phi)$ that drives the variation in $m_{e}$ in this model. We consider that the variations in $\phi$ are small and they do not produce significant contributions to the Friedmann equation. As we did in a previous work (Mosquera et al. 2008), we build a piecewise approximate solution by joining solutions obtained by keeping only certain terms of the Friedmann equation, which are relevant in the following domination regimes: (1) radiation and matter, and (2) matter and the cosmological constant. In this way, solution 1 can be applied to nucleosynthesis and the recombination of primordial hydrogen, whereas solution 2 is appropriate for quasar absorption systems, geophysical data, and atomic clocks.

Defining the variable $\vartheta$ as $d \vartheta=d \tau / a$, where $\tau=H_{0} t$ and $t$ is the cosmic time, the expression for the scale factor in the radiation and matter regime is

$$
a_{\mathrm{RM}}(\vartheta)=\frac{\Omega_{m}}{4} \vartheta^{2}+\sqrt{\Omega_{r}} \vartheta,
$$

and the relationship between $\tau$ and $\vartheta$ is

$$
\tau(\vartheta)=\frac{\Omega_{m}}{12} \vartheta^{3}+\frac{\sqrt{\Omega_{r}}}{2} \vartheta^{2} .
$$

The solution for the scale factor in the matter and cosmological constant regime can be written as

$$
\begin{aligned}
& a_{\mathrm{MC}}(\tau)= \\
& \quad\left(\frac{\Omega_{m}}{\Omega_{\Lambda}}\right)^{1 / 3}\left\{\sinh \left[\frac{3}{2} \sqrt{\Omega_{\Lambda}}\left(\tau-\tau_{0}\right)+\sinh ^{-1} \sqrt{\frac{\Omega_{\Lambda}}{\Omega_{\mathrm{m}}}}\right]\right\}^{2 / 3},
\end{aligned}
$$

\begin{tabular}{|c|c|c|c|}
\hline Redshift & $\Delta m_{e} /\left(m_{e}\right)_{0}{ }^{\mathrm{a}}$ & Redshift & $\Delta m_{e} /\left(m_{e}\right)_{0}^{\mathrm{a}}$ \\
\hline $0.24 \ldots \ldots \ldots \ldots \ldots$ & $1.21 \pm 2.10$ & $1.78 \ldots \ldots \ldots \ldots \ldots$. & $-2.59 \pm 0.90$ \\
\hline $0.31 \ldots \ldots \ldots \ldots \ldots$ & $-0.61 \pm 4.27$ & 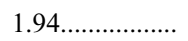 & $3.30 \pm 0.44$ \\
\hline $0.40 \ldots \ldots \ldots \ldots \ldots \ldots$ & $3.22 \pm 3.15$ & 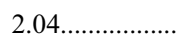 & $5.20 \pm 2.76$ \\
\hline 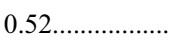 & $-2.95 \pm 1.05$ & $2.35 \ldots \ldots \ldots \ldots \ldots$ & $-2.54 \pm 1.82$ \\
\hline $0.52 \ldots \ldots \ldots \ldots \ldots \ldots$ & $0.26 \pm 3.67$ & & \\
\hline
\end{tabular}

\section{TABLE 5}

Absorption Redshift and Value of $\Delta m_{e} /\left(m_{e}\right)_{0}$ Obtained Comparing Radio and Molecular Redshifts (Tzanavaris et al. 2007)

\begin{tabular}{|c|c|c|c|}
\hline Frequencies & $\dot{m}_{e} /\left(m_{e}\right)_{0} \pm \sigma^{\mathrm{a}}$ & $\Delta t^{\mathrm{b}}$ & Reference \\
\hline $\mathrm{Hg}^{+}$and $\mathrm{Cs}$ & $0.2 \pm 7.0$ & 5 & Fischer et al. (2004) \\
\hline 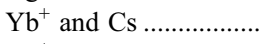 & $1.2 \pm 4.4$ & 2.8 & Peik et al. (2004) \\
\hline $\mathrm{Hg}^{+}$and $\mathrm{Cs}$ & $0.0 \pm 7.0$ & 2 & Bize et al. (2003) \\
\hline
\end{tabular}

\footnotetext{
${ }^{\text {a }}$ In units of $10^{-5}$.
}

TABLE 6

Compared Clocks and Value of $\dot{m}_{e} /\left(m_{e}\right)_{0}$

${ }^{\text {a }}$ In units of $10^{-15}$

b Time interval for which the variation was measured, in units of yr.

where $\tau_{0}=H_{0} t_{0}$ and $t_{0}$ is the age of the universe. To obtain the last solution we have considered that the scale factor must be a continuous and smooth function of time.

In the Barrow-Magueijo model, $m_{e}$ is controlled by a dilaton field $\phi$ defined by $m_{e}=\left(m_{e}\right)_{0} \exp (\phi)$, and variations in $m_{e}$ occur as soon as the universe cools down below the $m_{e}$ threshold. The minimal dynamics for $\phi$ is set by the kinetic Lagrangian

$$
\mathcal{L}_{\phi}=\frac{\omega}{2} \partial_{\mu} \phi \partial^{\mu} \phi
$$

where $\omega$ is a coupling constant. From this Lagrangian, the equation of motion of the scalar field can be derived as

$$
\left(\dot{\phi} a^{3}\right)=-M \exp [\phi]
$$

with $M \simeq \rho_{e 0} a_{0}^{3} c^{4} / \omega$. This is a second-order equation for $\phi$, with the boundary condition $\phi_{0}=0$. If the mass variations are small, $e^{\phi} \simeq 1$ can be set to obtain an analytical expression for $\phi$.

For convenience, we define $\beta=\frac{1}{4} \Omega_{m} \Omega_{r}^{-1 / 2}, \xi=\Omega_{m} \Omega_{r}^{-3 / 4} \Omega_{\Lambda}^{-1 / 4}$, $\gamma=\Omega_{\Lambda}^{1 / 2} \Omega_{m}^{-1 / 2}$, and

$$
\begin{aligned}
f(\xi) & =\frac{2+(\xi-2) \sqrt{1+\xi}}{\xi^{2}}, \\
C & =\sinh ^{-1} \xi^{-1 / 2}-f(\xi) .
\end{aligned}
$$

Provided $\phi=\ln \left(m_{e} /\left(m_{e}\right)_{0}\right) \simeq \Delta m_{e} /\left(m_{e}\right)_{0}$, the expressions for the variation in $m_{e}$ in the two regimes are as follows:

1. For $\tau<\tau_{1}$ (where $\tau_{1}$ is defined by $a_{\mathrm{RM}}\left(\tau_{1}\right)=a_{\mathrm{MC}}\left(\tau_{1}\right)=$ $\left(\Omega_{r} / \Omega_{\Lambda}\right)^{1 / 4}$; see Mosquera et al. 2008):

$$
\begin{aligned}
& \frac{\Delta m_{e}}{\left(m_{e}\right)_{0}}(\vartheta)= \\
& \frac{2}{3} \frac{M}{H_{0}^{2}} \frac{1}{\Omega_{m}}\left\{-2 \ln \left[\frac{2(\beta \vartheta+1)}{1+\sqrt{1+\xi}}\right]+\frac{1}{\beta \vartheta+1}-\frac{2}{1+\sqrt{1+\xi}}\right. \\
& \left.+\frac{2}{3} f(\xi) \sqrt{1+\xi}+\frac{1}{4} \ln \left(\frac{\Omega_{\Lambda}}{\Omega_{r}}\right)-\frac{2}{3}\left(\sinh ^{-1} \gamma-C\right) \frac{\sqrt{1+\gamma^{2}}}{\gamma}\right\} \\
& +\frac{A}{H_{0}} \frac{\Omega_{m}}{\Omega_{r}^{3 / 2}}\left\{\frac{1}{2} \ln \left(\frac{\beta \vartheta+1}{\beta \vartheta}\right)+\frac{1}{2} \ln \left(\frac{\sqrt{1+\xi}-1}{\sqrt{1+\xi}+1}\right)-\frac{1}{4 \beta \vartheta}\right. \\
& \left.-\frac{1}{4(\beta \vartheta+1)}+\frac{[\xi-(2 / 3)] \sqrt{1+\xi}+(2 / 3)\left(\sqrt{1+\gamma^{2}} / \gamma\right)}{\xi^{2}}\right\} .
\end{aligned}
$$

The relationship between $\tau$ and $\vartheta$ is given by equation (3). 
TABLE 7

Best-Fit Parameters of the Model

\begin{tabular}{cccc}
\hline \hline$M / H_{0}^{2}$ & $A / H_{0}$ & $G \omega / c^{4}$ & $\chi_{\min }^{2} /(N-2)$ \\
\hline$-7.30_{-2.02}^{+2.10}$ & $3.60_{-1.50}^{+1.44}$ & $-0.336_{-0.093}^{+0.097}$ & 1.14 \\
\hline
\end{tabular}

Noте.-The table does not include entry 7 of Table 5 . The value for the $M / H_{0}^{2}$ parameter is in units of $10^{-6}$, and the value for the $A / H_{0}$ parameter is in units of $10^{-13}$.

$$
\begin{aligned}
& \text { 2. For } \tau>\tau_{1} \text { : } \\
& \frac{\Delta m_{e}}{\left(m_{e}\right)_{0}}(\tau) \\
& =\phi_{0}+\frac{M}{H_{0}^{2}} \frac{2}{3 \Omega_{m}}\left(\sqrt{\Omega_{\Lambda}} \tau \operatorname{coth}\left(C+\frac{3}{2} \sqrt{\Omega_{\Lambda}} \tau\right)\right. \\
& -\frac{2}{3} \ln \left[\sinh \left(C+\frac{3}{2} \sqrt{\Omega_{\Lambda}} \tau\right)\right] \\
& \left.+\frac{2}{3}\left\{\ln \gamma-\frac{\sqrt{1+\gamma^{2}}}{\gamma}\left[C+\ln \left(\gamma+\sqrt{1+\gamma^{2}}\right)\right]\right\}\right) \\
& +\frac{A}{H_{0}} \frac{2 \sqrt{\Omega_{\Lambda}}}{3 \Omega_{m}}\left[-\operatorname{coth}\left(C+\frac{3}{2} \sqrt{\Omega_{\Lambda}} \tau\right)+\frac{\sqrt{1+\gamma^{2}}}{\gamma}\right]
\end{aligned}
$$

where $A$ is an integration constant.

\section{RESULTS}

The model described in $\S 7$ predicts the variation in $m_{e}$ as a function of time and has two independent dimensionless parameters, $M / H_{0}^{2}$ and $A / H_{0}$. We do not fix $A / H_{0}$ to zero, as previous works did (Barrow \& Magueijo 2005). To constrain these parameters, we use the data described in the previous sections. We perform a $\chi^{2}$ test to obtain the best-fit parameters of the model. In order to obtain the parameters consistently with our assumption that the energy density of the field $\phi\left[\epsilon_{\phi}=\left(1 / c^{2}\right)(\omega / 2) \dot{\phi}^{2}\right]$ can be neglected in the Friedmann equation, we add to the $\chi^{2}$ expression, a term that controls the contribution of $\phi$ to the Friedmann equation to make it less important than the radiation term right after $m_{e}$ threshold is crossed.

The result of the statistical analysis shows that there is no good fit for the whole data set. We repeated the analysis excluding one group of data at a time. We found that reasonable fits can be obtained excluding the quasar at $z=1.94$ (see Table 5) and that the data from nucleosynthesis is crucial to determine the value of $A / H_{0}$. Otherwise, the group of data from Table 4 is important to determine the value of $M / H_{0}^{2}$. The results are shown in Table 7.

Since the Barrow-Magueijo model is written in terms of the coupling constant $\omega$, we derive its best value from the previous constraints. Since $M \simeq \rho_{e 0} a_{0}^{3} c^{4} / \omega$ we obtain the following relationship:

$$
\frac{G \omega}{c^{4}}=\frac{3}{8 \pi}\left(1-\frac{f_{\mathrm{He}}}{2}\right) \Omega_{b} \frac{m_{e}}{m_{p}}\left(\frac{M}{H_{0}^{2}}\right)^{-1}
$$

where $f_{\mathrm{He}}$ is the fraction of the total number of baryons in the form of He and can be written as a function of the total observed mass abundance of $\mathrm{He}\left(M_{\mathrm{He}} / M_{\mathrm{H}}\right)$. According to the values of $M / H_{0}^{2}$ from Table 7 and using $f_{\mathrm{He}}=0.19$ (taking $M_{\mathrm{He}} / M_{\mathrm{H}}=$
0.24 ), we obtain the bounds on the dimensionless quantity $G \omega / c^{4}$ presented in Table 7.

\section{SUMMARY AND CONCLUSIONS}

In this paper we have put limits on the time variation in the electron mass at primordial nucleosynthesis time using observational primordial abundances of $\mathrm{D},{ }^{4} \mathrm{He}$, and ${ }^{7} \mathrm{Li}$, and we have analyzed in detail the consequences of considering different groups of data. We have also considered the variations in $\langle v\rangle$ during $\mathrm{BBN}$ and analyzed the differences with the variations in $m_{e}$ during the same epoch. In addition, we have used the 3 year data from the cosmic microwave background and the final 2dFGRS power spectrum to obtain bounds on the variation in $m_{e}$ at recombination and an estimation of the cosmological parameters. Together with other bounds on the variation in the late universe, which come from quasar absorption systems, the half-life of longlived $\beta$-decayers, and atomic clocks, we put constraints on the Barrow-Magueijo model for the variation in $m_{e}$. We have improved the solutions by taking into account the detailed evolution of the scale factor and the complete solution for the scalar field that drives the variation in $m_{e}$.

In the original paper (Barrow \& Magueijo 2005) some approximations in the evolution of the scale factor are assumed, with a consequent simplification in the solution for the scalar field. Another improvement of our derivation is that we have not neglected the first integration constant $A$ which plays an important role in the solution of the scalar field at early times. In fact, integrating equation (6) once, we can write

$$
\dot{\phi} a^{3}=-M\left(t-\frac{A}{M}\right)=-M\left(t+8.47 \times 10^{2} \mathrm{yr}\right),
$$

where we have used the best-fit values for the parameters $M / H_{0}^{2}$ and $A / H_{0}$, and $h=0.73$. Note that the second term in the righthand side of equation (11) is dominant in the early universe, in particular, during nucleosynthesis.

Barrow \& Magueijo (2005) presented a bound of $G|\omega|>0.2$ (with $c=1$ ). They obtained this constraint using bounds from quasars at $z \sim 1$, whereas we use all the available bounds on the variation in $m_{e}$ at different cosmological times. In Appendix C we briefly discuss the difference in both analysis. From data supporting the weak equivalence principle, they obtain $G|\omega|>10^{3}$, while we obtain $-0.615<G \omega / c^{4}<-0.045$ (3 $\sigma$ interval $)$ using data from different cosmological timescales. More research both on time variation data and on the bound from WEP is needed to clarify this discrepancy.

Finally, we remark that, at $2 \sigma$, the value of $\omega$ is negative. This should not be surprising. Indeed, negative kinetic terms in the Lagrangian have already been considered in $k$-essence models with a phantom energy component (Caldwell 2002).

Support for this work was provided by project G11/G071, UNLP, and PIP 5284 CONICET. The authors would like to thank Andrea Barral, Federico Bareilles, Alberto Camyayi, and Juan Veliz for technical and computational support. The authors would also like to thank Ariel Sanchez for support with CosmoMC. M. E. M. wants to thank Sergio Iguri for the helpful discussions. C. G. S. gives special thanks to Licia Verde and Nelson Padilla for useful discussion. S. J. L. wants to thank Luis Chimento for useful discussions. 


\section{APPENDIX A}

\section{PHYSICS AT BBN}

In this appendix we discuss the dependences of the physical quantities involved in the calculation of the abundances of the light elements with $m_{e}$ and variation in the Higgs vacuum expectation value. We also discuss how these quantities are changed in the Kawano code.

\section{A1. VARIATION IN THE ELECTRON MASS}

A change in the value of $m_{e}$ at the time of primordial nucleosynthesis with respect to its present value affects derived physical quantities, such as the sum of the electron and positron energy densities, the sum of the electron and positron pressures, and the difference of the electron and positron number densities. In Kawano's code, these quantities are calculated as follows:

$$
\begin{gathered}
\rho_{e^{-}}+\rho_{e^{+}}=\frac{2}{\pi^{2}} \frac{\left(m_{e} c^{2}\right)^{4}}{(\hbar c)^{3}} \sum_{n}(-1)^{n+1} \cosh \left(n \phi_{e}\right) M(n z), \\
\frac{p_{e^{-}}+p_{e^{+}}}{c^{2}}=\frac{2}{\pi^{2}} \frac{\left(m_{e} c^{2}\right)^{4}}{(\hbar c)^{3}} \sum_{n} \frac{(-1)^{n+1}}{n z} \cosh \left(n \phi_{e}\right) N(n z), \\
\frac{\pi^{2}}{2}\left(\frac{\hbar c^{3}}{m_{e} c^{2}}\right)^{3} z^{3}\left(n_{e^{-}}-n_{e^{+}}\right)=z^{3} \sum_{n}(-1)^{n+1} \sinh \left(n \phi_{e}\right) L(n z),
\end{gathered}
$$

where $z=m_{e} c 2 / k T_{\gamma}, \phi_{e}$ is the electron chemical potential and $L(z), M(z)$, and $N(z)$ are related to the modified Bessel function (Kawano 1988, 1992). In order to include the variation in $m_{e}$ we replace, in all three equations, $m_{e}$ by $\left(m_{e}\right)_{0}\left[1+\Delta m_{e} /\left(m_{e}\right)_{0}\right]$. The change in these quantities, due to a change in $m_{e}$, affects their derivatives and the expansion rate through the Friedmann equation.

The $n \leftrightarrow p$ reaction rates and the other weak decay rates are changed if $m_{e}$ varies with time. The total $n \rightarrow p$ reaction rate is calculated by

$$
\lambda=K \int_{m_{e}}^{\infty} \frac{d E_{e} E_{e} p_{e}\left(E_{e}+\Delta m_{\mathrm{np}}\right)^{2}}{\left(1+e^{E_{e} / T_{\gamma}}\right)\left(1+e^{-\left(E_{e}+\Delta m_{\mathrm{np}}\right) / T_{\nu}-\xi_{l}}\right)}+K \int_{m_{e}}^{\infty} \frac{d E_{e} E_{e} p_{e}\left(E_{e}-\Delta m_{\mathrm{np}}\right)^{2}}{\left(1+e^{-E_{e} / T_{\gamma}}\right)\left(1+e^{\left(E_{e}-\Delta m_{\mathrm{np}}\right) / T_{\nu}-\xi_{l}}\right)}
$$

where $E_{e}$ and $p_{e}$ are the electron energy and momentum, respectively, $\Delta m_{\mathrm{np}}$ is the neutron-proton mass difference, $K$ is a normalization constant proportional to $G_{F}^{2}$, and $E_{e}=\left(p_{e}^{2}+m_{e}^{2}\right)^{1 / 2}$.

It is worth mentioning that the most important changes in the primordial abundances (due to a change in $m_{e}$ ) result from the change in the weak rates rather than the change in the expansion rate (Yoo \& Scherrer 2003).

\section{A2. VARIATION IN THE HIGGS VACUUM EXPECTATION VALUE}

If the value of $\langle v\rangle$ during $\mathrm{BBN}$ is different than its present value, the electron mass, the Fermi constant, the neutron-proton mass difference, and the deuterium binding energy take different values than the current ones. The electron mass is proportional to the Higgs vacuum expectation value.

The Fermi constant is proportional to $\langle v\rangle^{-2}$ (Dixit \& Sher 1988). This dependence affects the $n \leftrightarrow p$ reaction rates, since $K \sim G_{F}^{2}$. The neutron-proton mass difference changes by (Christiansen et al. 1991)

$$
\frac{\delta \Delta m_{\mathrm{np}}}{\Delta m_{\mathrm{np}}}=1.587 \frac{\Delta\langle v\rangle}{\langle v\rangle_{0}}
$$

affecting $n \leftrightarrow p$ reaction rates (see eq. [A4]) and the initial neutrons and protons abundances:

$$
Y_{n}=\frac{1}{1+e^{\Delta m_{\mathrm{np}} / T_{9}+\xi}}, Y_{p}=\frac{1}{1+e^{-\Delta m_{\mathrm{np}} / T_{9}-\xi}},
$$

where $T_{9}$ is the temperature in units of $10^{9} \mathrm{~K}$. In order to include these effects we replace $\Delta m_{\mathrm{np}}$ by $\Delta m_{\mathrm{np}}\left(1+\delta \Delta m_{\mathrm{np}} / \Delta m_{\mathrm{np}}\right)$. The deuterium binding energy must also be corrected by $\Delta \epsilon_{\mathrm{D}} / \epsilon_{\mathrm{D}}=k\left(\Delta\langle v\rangle /\langle v\rangle_{0}\right)$, where $k$ is a model-dependent constant. In this work, following Chamoun et al. (2007), we assume $k=-0.045$. This correction affects the initial value of the deuterium abundance.

Once again we replace $\epsilon_{\mathrm{D}}$ by $\epsilon_{\mathrm{D}}\left(1+\Delta \epsilon_{\mathrm{D}} / \epsilon_{\mathrm{D}}\right)$ in the code. 


\section{APPENDIX B}

\section{PHYSICS AT THE RECOMBINATION EPOCH}

During the recombination epoch the ionization fraction $x_{e}$ is determined by the balance between photoionization and recombination. The recombination equation is

where

$$
-\frac{d}{d t}\left(\frac{n_{e}}{n}\right)=C\left(\frac{\alpha_{c} n_{e}^{2}}{n}-\beta_{c} \frac{n_{1 s}}{n} e^{-\left(B_{1}-B_{2}\right) / k T}\right)
$$

$$
C=\frac{\left(1+K \Lambda_{2 s, 1 s} n_{1 s}\right)}{\left[1+K\left(\beta_{c}+\Lambda_{2 s, 1 s}\right) n_{1 s}\right]}
$$

is the Peebles factor, which inhibits the recombination rate due to the presence of Ly $\alpha$ photons. The redshift of these photons is $K=\lambda_{\alpha}^{3} a / 8 \pi \dot{a}$, with $\lambda_{\alpha}=8 \pi c / 3 B_{1}$, and $\Lambda_{2 s, 1 s}$ is the rate of decay of the $2 s$ excited state to the ground state via two-photon emission, and scales as $m_{e}$. Recombination directly to the ground state is strongly inhibited, so case B recombination takes place. The case $\mathrm{B}$ recombination coefficient $\alpha_{c}$ is proportional to $m_{e}^{-3 / 2}$. The photoionization coefficient depends on $\alpha_{c}$, but it also has an additional dependence on $m_{e}$,

$$
\beta_{c}=\alpha_{c}\left(\frac{2 \pi m_{e} k T_{m}}{h^{2}}\right)^{3 / 2} e^{-B_{2} / k T_{m}}
$$

The most important effects of a change in $m_{e}$ during recombination would be due to its influence on the Thomson scattering cross section $\sigma_{\mathrm{T}}=\left(8 \pi \hbar^{2} c^{2} / 3 m_{e}^{2}\right) \alpha^{2}$ and the binding energy of hydrogen $B_{1}=\frac{1}{2} \alpha^{2} m_{e} c^{2}$.

\section{APPENDIX C}

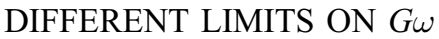

In this appendix we compare the limits obtained by Barrow \& Magueijo (2005) on $G \omega$ with our bounds. We stress that we have performed a $\chi^{2}$ test using all available observational and experimental data, while Barrow \& Magueijo (2005) consider $|\Delta \mu / \mu|<10^{-5}$ for data at redshift of order 1 . Moreover, most exact individual bounds from quasar absorption systems are not consistent with null variation at least at $1 \sigma$.

Let us consider for example the last entry of Table 4: $-3.5 \times 10^{-5}<\Delta \mu / \mu<-0.72 \times 10^{-5}$. Using the same approximation as Barrow \& Magueijo (2005) we find that $-0.28<G \omega<-0.05$ for this measurement, which is of the same order of magnitude as obtained considering all data.

\section{REFERENCES}

Asplund, M., Lambert, D. L., Nissen, P. E., Primas, F., \& Smith, V. V. 2006, ApJ, 644, 229

Barger, V., Kneller, J. P., Langacker, P., Marfatia, D., \& Steigman, G. 2003, Phys. Lett. B, 569, 123

Barr, S. M., \& Mohapatra, P. K. 1988, Phys. Rev. D, 38, 3011

Barrow, J. D., \& Magueijo, J. 2005, Phys. Rev. D, 72, 043521

Barrow, J. D., Sandvik, H. B., \& Magueijo, J. 2002, Phys. Rev. D, 65, 063504

Bekenstein, J. D. 1982, Phys. Rev. D, 25, 1527

- 2002, Phys. Rev. D, 66, 123514

Bergström, L., Iguri, S., \& Rubinstein, H. 1999, Phys. Rev. D, 60, 45005

Bize, S., et al. 2003, Phys. Rev. Lett., 90, 150802

Boesgaard, A. M., Novicki, M. C., \& Stephens, A. 2005, in IAU Symp. 228, From Lithium to Uranium: Elemental Tracers of Early Cosmic Evolution, ed.

V. Hill, P. Francois, \& F. Primas (Cambridge: Cambridge Univ. Press), 29

Bonifacio, P., \& Molaro, P. 1997, MNRAS, 285, 847

Bonifacio, P., et al. 2002, A\&A, 390, 91

- 2007, A\&A, 462, 851

Brax, P., van de Bruck, C., Davis, A. C., \& Rhodes, C. S. 2003, Ap\&SS, 283, 627

Burles, S., \& Tytler, D. 1998a, ApJ, 499, 699 1998b, ApJ, 507, 732

Caldwell, R. R. 2002, Phys. Lett. B, 545, 23

Chamoun, N., Landau, S. J., Mosquera, M. E., \& Vucetich, H. 2007, J. Phys. G, 34, 163

Christiansen, H. R., Epele, L. N., Fanchiotti, H., \& García Canal, C. A. 1991, Phys. Lett. B, 267, 164

Cole, S., et al. 2005, MNRAS, 362, 505

Crighton, N. H. M., Webb, J. K., Ortiz-Gil, A., \& Fernández-Soto, A. 2004, MNRAS, 355, 1042

Damour, T., Piazza, F., \& Veneziano, G. 2002a, Phys. Rev. Lett., 89, 081601 2002b, Phys. Rev. D, 66, 046007
Damour, T., \& Polyakov, A. M. 1994, Nucl. Phys. B, 95, 10347

Dixit, V. V., \& Sher, M. 1988, Phys. Rev. D, 37, 1097

Fischer, M., et al. 2004, Phys. Rev. Lett., 92, 230802

Freedman, W. L., et al. 2001, ApJ, 553, 47

Gleiser, M., \& Taylor, J. G. 1985, Phys. Rev. D, 31, 1904

Ivanchik, A., Petitjean, P., Rodriguez, E., \& Varshalovich, D. 2003, Ap\&SS, 283,583

Ivanchik, A., Petitjean, P., Varshalovich, D., Aracil, B., Srianand, R., Chand, H., Ledoux, C., \& Boissé, P. 2005, A\&A, 440, 45

Izotov, Y. I., Thuan, T. X., \& Stasińska, G. 2007, ApJ, 662, 15

Jones, W. C., et al. 2006, ApJ, 647, 823

Kaluza, T. 1921, Sitzungber. Preussischer Akad. Wiss. K, 1, 966

Kawano, L. 1988, Let's Go: Early Universe. Guide to Primordial Nucleosynthesis Programming (FERMILAB-PUB-88-034-A; Batavia: Fermilab), http://lss .fnal.gov/archive/1988/pub/Pub-88-034-A.pdf

. 1992, Let's Go: Early Universe. II. Primordial Nucleosynthesis: The Computer Way (FERMILAB-PUB-92-004-A; Batavia: Fermilab), http://lss .fnal.gov/archive/1992/pub/Pub-92-004-A.pdf

Kirkman, D., Tytler, D., Suzuki, N., O’Meara, J. M., \& Lubin, D. 2003, ApJS, 149, 1 Klein, O. 1926, Z. Phys., 37, 895

Kujat, J., \& Scherrer, R. J. 2000, Phys. Rev. D, 62, 023510

Kuo, C.-L., et al. 2004, ApJ, 600, 32

Levshakov, S. A., Dessauges-Zavadsky, M., D’Odorico, S., \& Molaro, P. 2002, MNRAS, 333, 373

Lewis, A., \& Bridle, S. 2002, Phys. Rev. D, 66, 103511

Lewis, A., Challinor, A., \& Lasenby, A. 2000, ApJ, 538, 473

Maeda, K. 1988, Mod. Phys. Lett. A, 31, 243

Molaro, P., Bonifacio, P., \& Pasquini, L. 1997, MNRAS, 292, L1

Mosquera, M. E., Scóccola, C. G., Landau, S. J., \& Vucetich, H. 2008, A\&A, 478,675 
Olive, K. A., Pospelov, M., Qian, Y.-Z., Manhès, G., Vangioni-Flam, E., Coc, A., \& Cassé, M. 2004, Phys. Rev. D, 69, 027701

Oliveira, C. M., Moos, H. W., Chayer, P., \& Kruk, J. W. 2006, ApJ, 642, 283

O’Meara, J. M., Burles, S., Prochaska, J. X., Prochter, G. E., Bernstein, R. A., \& Burgess, K. M. 2006, ApJ, 649, L61

O’Meara, J. M., Tytler, D., Kirkman, D., Suzuki, N., Prochaska, J. X., Lubin, D., \& Wolfe, A. M. 2001, ApJ, 552, 718

Overduin, J. M., \& Wesson, P. S. 1997, Phys. Rep., 283, 303

Palma, G. A., Brax, P., Davis, A. C., \& van de Bruck, C. 2003, Phys. Rev. D, 68,123519

Peik, E., Lipphardt, B., Schnatz, H., Schneider, T., Tamm, C., \& Karshenboim, S. G. 2004, Phys. Rev. Lett., 93, 170801

Peimbert, M., Luridiana, V., \& Peimbert, A. 2007, ApJ, 666, 636

Pettini, M., \& Bowen, D. V. 2001, ApJ, 560, 41

Piacentini, F., et al. 2006, ApJ, 647, 833

Potekhin, A. Y., Ivanchik, A. V., Varshalovich, D. A., Lanzetta, K. M., Baldwin, J. A., Williger, G. M., \& Carswell, R. F. 1998, ApJ, 505, 523

Raftery, A. E., \& Lewis, S. M. 1992, in Bayesian Statistics, ed J. M. Bernado (Oxford: Oxford Univ. Press), 765

Readhead, A. C. S., et al. 2004, ApJ, 609, 498

Ryan, S. G., Beers, T. C., Olive, K. A., Fields, B. D., \& Norris, J. E. 2000, ApJ, 530, L57
Sánchez, A. G., Baugh, C. M., Percival, W. J., Peacock, J. A., Padilla, N. D., Cole, S., Frenk, C. S., \& Norberg, P. 2006, MNRAS, 366, 189

Seager, S., Sasselov, D. D., \& Scott, D. 1999, ApJ, 523, L1

Sisterna, P., \& Vucetich, H. 1990, Phys. Rev. D, 41, 1034

Spergel, D. N., et al. 2003, ApJS, 148, 175

. 2007, ApJS, 170, 377

Steigman, G. 2005, Phys. Scr. T, 121, 142

. 2006, Int. J. Mod. Phys. E, 15, 1

Tzanavaris, P., Murphy, M. T., Webb, J. K., Flambaum, V. V., \& Curran, S. J. 2007, MNRAS, 374, 634

Varshalovich, D. A., \& Levshakov, S. A. 1993, Soviet J. Exp. Theor. Phys. Lett., 58, 237

Villa, F., Mandolesi, N., \& Butler, R. C. 2003, Mem. Soc. Astron. Italiana, 74, 223

Weinberg, S. 1983, Phys. Lett. B, 125, 265

White, M. 2006, NewA Rev., 50, 938

Wu, Y., \& Wang, Z. 1986, Phys. Rev. Lett., 57, 1978

Yoo, J. J., \& Scherrer, R. J. 2003, Phys. Rev. D, 67, 043517

Youm, D. 2001a, Phys. Rev. D, 63, 125011

. 2001b, Phys. Rev. D, 64, 085011 